\title{
The "Three-Squares" Theorem for Continuous Functions
}

\author{
C. A. Berenstein \& B. A. Taylor \\ Communicated by M.M. SCHIFFER
}

\section{Introduction}

Let $\mathscr{P}$ be a family of compact subsets of the Euclidean plane $\mathbb{R}^{2}$, and let $\mathscr{I}$ denote the group of all translations of $\mathbb{R}^{2}$. We shall consider here the Pompeiu problem for the family $\mathscr{P}$, namely, to determine when the only continuous function $f$ on $\mathbb{R}^{2}$ such that

$$
\int_{\tau(P)} f(x) d x=0 \quad \text { for all } \tau \in \mathscr{I} \text { and } P \in \mathscr{P}
$$

is the zero function. (Here $d x$ denotes Lebesgue measure on $\mathbb{R}^{2}$.) In this note we solve this problem when the family $\mathscr{P}$ consists of rectangles. In particular, we will prove the following result.

Theorem 1. Suppose $\mathscr{P}$ is a finite family of squares with sides parallel to the coordinate axes. Then $f \equiv 0$ is the only continuous solution of equation (1) if and only if $\mathscr{P}$ contains three squares of side length $a_{1}, a_{2}, a_{3}$ such that $a_{1} / a_{2}, a_{2} / a_{3}, a_{3} / a_{1}$ are irrational.

Thus for "most" choices of three squares $P_{1}, P_{2}, P_{3}$ the validity of (1) for $\mathscr{P}=\left\{P_{1}, P_{2}, P_{3}\right\}$ implies $f \equiv 0$.

The Pompeiu problem has an extensive history which we will not discuss here. The reader is referred to the paper [13] of L. ZaLCMAN for an excellent account of the problem, including the "two-circles theorem" of Delsarte for harmonic functions, which is the motivation for our Theorem 1. We do mention, however, the following equivalent formulation of the Pompeiu problem, which is known as the Morera problem: Suppose all the sets $P$ of the family $\mathscr{P}$ have "nice" boundaries (say piecewise smooth, so that the Green theorem can be applied to change integrations from $P$ to $\partial P$ ). When is it true that the only continuous functions $f$ such that

$$
\int_{\tau(\partial P)} f(z) d z=0 \quad \text { for all } \tau \in \mathscr{I} \text { and } P \in \mathscr{P}
$$

are entire analytic functions (here $d z=d x+i d y$ is the usual complex differential)? We refer to [1], Section 4, especially Theorem 4.1, or to [13] for the proof of the equivalence of the Pompeiu and Morera problems. (Note that the Pompeiu and Morera problems in [1] are formulated in the different (and nonequivalent) 
form in which the group $\mathscr{I}$ of translations of $\mathbb{R}^{2}$ is replaced by the larger group $\Sigma$ of all rigid motions of $\mathbb{R}^{2}$. This does not however, change the equivalence of the Pompeiu and Morera problems in the form stated here.) Thus the following theorem is a consequence of Theorem 1 .

Theorem 2. Suppose $\mathscr{P}$ is a finite family of squares with sides parallel to the coordinate axes. Then solutions of (2) must be entire analytic functions if and only if $\mathscr{P}$ contains three squares of side length $a_{1}, a_{2}, a_{3}$ such that $a_{1} / a_{2}, a_{2} / a_{3}, a_{3} / a_{1}$ are irrational.

The Pompeiu problem is a special case of the spectral synthesis problem formulated by L. SCHWARTZ in [12]. To state this problem, note first that if $\chi_{P}$ is the characteristic function of the set $P \in \mathscr{P}$, and $f(x)=f(-x)$, then (1) can be written as the system of homogeneous convolution equations

$$
\left(f * \chi_{P}\right)(y)=\int_{P} f(x-y) d x=0 \quad \text { for all } y \in \mathbb{R}^{2} \text { and } \quad P \in \mathscr{P} .
$$

It is easy to see (by smoothing $f$ ) that the system of equations (3) has a nontrivial solution $f$ if and only if there exists a nontrivial solution $f$ in the space $\mathscr{E}\left(\mathbb{R}^{2}\right)$ of all infinitely differentiable functions on $\mathbb{R}^{2}$. The spectral synthesis problem of SCHWARTZ for a collection $\mathscr{U}$ of distributions of compact support on $\mathbb{R}^{n}$ (i.e. $\left.\mathscr{U} \subset \mathscr{E}^{\prime}\left(\mathbb{R}^{n}\right)\right)$ is to show that each function $f \in \mathscr{E}\left(\mathbb{R}^{n}\right)$ satisfying

$$
f * \mu=0 \quad \text { for all } \mu \in \mathscr{U}
$$

must be a limit in $\mathscr{E}\left(\mathbb{R}^{n}\right)$ of linear combinations of exponential-polynomial solutions of (4), that is solutions of the form

$$
p(x) \exp (i z \cdot x)=p(x) \exp \left[i\left(z_{1} \cdot x_{1}+\cdots+z_{n} \cdot x_{n}\right)\right]
$$

where $z=\left(z_{1}, \ldots, z_{n}\right) \in \mathbb{C}^{n}$ and $p$ is a polynomial. In particular, if spectral synthesis holds for the collection $\mathscr{U}$, then (4) has a nontrivial solution if and only if there is an exponential solution of (4). Equivalently, if

$$
\hat{\mu}(z)=\mu(\exp (i z \cdot x))
$$

is the Fourier transform of $\mu \in \mathscr{E}^{\prime}\left(\mathbb{R}^{n}\right)$, then we readily check that (4) has an exponential solution if and only if the functions $\{\hat{\mu}(z): \mu \in \mathscr{U}\}$ have a common zero. If $P \subset \mathbb{R}^{2}$ is a rectangle with center at $(0,0)$ and sides parallel to the coordinate axes of length $2 \mathrm{a}, 2 \mathrm{~b}$, respectively, then

$$
\hat{\chi}_{P}\left(z_{1}, z_{2}\right)=\int_{-b}^{b} \int_{-a}^{a} e^{i\left(z_{1} x_{1}+z_{2} x_{2}\right)} d x_{1} d x_{2}=4 \frac{\sin a z_{1} \sin b z_{2}}{z_{1} z_{2}} .
$$

Thus, the common zeros of $\hat{\chi}_{P}, P \in \mathscr{P}$, are easy to compute if $\mathscr{P}$ is a family of such rectangles, and this provides a proof of the "only if" part of Theorem 1 . In fact, the condition " $a_{i} / a_{j}$ irrational" in Theorem 1 , is clearly equivalent to the assertion that the $\hat{\chi}_{P}, P \in \mathscr{P}$, have no common zeros in $\mathbb{C}^{2}$. Thus, the "if" part of Theorem 1 is proved provided we show that spectral synthesis holds for any collection $\mathscr{U} \subset \mathscr{E}^{\prime}\left(\mathbb{R}^{2}\right)$ which contains a distribution $\mu$ of compact support of the form $\mu=\chi_{P}$, where $P$ is a rectangle $\left(\mu=\chi_{P}\right.$ means $\mu(f)=\int_{P} f(x) d x$ for $\left.f \in \mathscr{E}\left(\mathbb{R}^{2}\right)\right)$. 
SCHWARTZ proved in [12] that every collection $\mathscr{U}$ of distributions of compact support in $\mathbb{R}^{1}$ (i.e. $\mathscr{U} \subset \mathscr{E}^{\prime}\left(\mathbb{R}^{1}\right)$ ) admits spectral synthesis. For $\mathscr{U} \subset \mathscr{E}^{\prime}\left(\mathbb{R}^{n}\right), n>1$, various special cases have been proved, see e.g. [1], [2], [3], [7], [9], [10], [13], [14]. However, D.I. GuREvich [4] recently produced a nice example which shows that, in general, spectral synthesis fails when $n>1$. Thus, the positive results which can be obtained must require special hypotheses on the family $\mathscr{U}$.

To prove our positive result, we will use the well-known equivalence of the spectral synthesis problem with the problem of closed ideals in the algebra $\mathscr{E}^{\prime}\left(\mathbb{R}^{n}\right)=\left\{\hat{\mu}(z): \mu \in \mathscr{E}^{\prime}\left(\mathbb{R}^{n}\right)\right\}$. By the Paley-Wiener-Schwartz Theorem, $\hat{\mathscr{E}}^{\prime}\left(\mathbb{R}^{n}\right)$ is identified as the space of all entire functions $F$ on $\mathbb{C}^{n}$ such that

$$
|F(z)| \leqq A(1+|z|)^{B} \exp (C|\operatorname{Im} z|),
$$

where $A, B, C$ are constants (depending on $F)$ and $\operatorname{Im} z=\left(\operatorname{Im} z_{1}, \ldots, \operatorname{Im} z_{n}\right) \in \mathbb{R}^{n}$ is the imaginary part of $z$. The space $\hat{\mathscr{E}}^{\prime}\left(\mathbb{R}^{n}\right)$ carries a natural topology (see e.g. [2], Chapter 5). For an ideal $I \subset \hat{\mathscr{E}}^{\prime}\left(\mathbb{R}^{n}\right)$, let $I_{\text {loc }}$ denote the ideal of functions in $\hat{\mathscr{E}}^{\prime}\left(\mathbb{R}^{n}\right)$ which are locally in $I$, i.e. $F \in I_{\text {loc }}$ if $F \in \mathscr{E}^{\prime}\left(\mathbb{R}^{n}\right)$ and, for each $z \in \mathbb{C}^{n}$, the germ of $F$ at $z$ belongs to the ideal generated by the germs of the functions in $I$ in the local ring $\mathcal{O}_{z}$ of germs of holomorphic functions at $z$ (c.f. [5], Chapter 6) Clearly, $I \subset I_{\mathrm{loc}}$. The closed ideals problem, for which the example of GUREVICH provides a counterexample, is to show that

$$
I \text { closed } \Leftrightarrow I=I_{\mathrm{loc}} .
$$

If $I=I(\mathscr{U}) \subset \widehat{\mathscr{E}}\left(\mathbb{R}^{n}\right)$ is the ideal generated by $\{\hat{\mu}: \mu \in \mathscr{U}\}$ for a collection $\mathscr{U} \subset \mathscr{E}^{\prime}\left(\mathbb{R}^{n}\right)$, then it is true that

$$
\text { spectral synthesis holds for } \mathscr{U} \Leftrightarrow I(\mathscr{U})=I(\mathscr{U})_{\mathrm{loc}} \text {. }
$$

We refer to [1], Proposition 2.4, for a discussion and proof of this equivalence.

Finally, we are able to state our main result.

Theorem 3. Let I be a closed ideal in $\mathscr{E}^{\prime}\left(\mathbb{R}^{2}\right)$. If I contains a function $F$ which is not identically zero, but whose zero set in $\mathbb{C}^{2}$ is a union of complex lines, then $I=I_{\mathrm{loc}}$.

In view of the equivalence (6), the observation following (4), and the computation (5) of the Fourier transform of the characteristic function of a rectangle, Theorem 3 implies the "if" part of Theorem 1 . Theorem 3 also implies that when the family $\mathscr{P}$ in equations (1) contains one product set, then there is a nontrivial solution of the equation (1) if and only if the Fourier transforms $\hat{\chi}_{P}\left(z_{1}, z_{2}\right)$, $P \in \mathscr{P}$, have a common zero in $\mathbb{C}^{2}$.

In Section 2 of the paper, we will give the proof of Theorem 3. At the end of the section, we also remark on the analogous theorem for $n \geqq 3$.

\section{Proof of Theorem 3}

The proof consists of several lemmas, each of which allows a reduction of the problem to the (known) one-variable case or carries out an argument used in the one-variable case. We will change notation slightly and denote the function $F$ 
in Theorem 3 by $f$. Write the hyperplanes in the zero set of $f$ as

$$
\theta_{j} \cdot z=c_{j}
$$

where $\theta_{j}=\left(\theta_{j 1}, \theta_{j 2}\right) \in \mathbb{C}^{2}, c_{j} \in \mathbb{C},\left|\theta_{j}\right|^{2}=\left|\theta_{j 1}\right|^{2}+\left|\theta_{j 2}\right|^{2}$, and $\theta_{j} \cdot z=\theta_{j 1} z_{1}+\theta_{j 2} z_{2}$. We may assume $f(0) \neq 0$; i.e. $c_{j} \neq 0$, by translating coordinates if necessary. Define

$$
\ell_{j}(z)=1-\left(\theta_{j} \cdot z\right) / c_{j}
$$

Thus each $\theta_{j}(z)$ divides $f$ in $\hat{\mathscr{E}}^{\prime}\left(\mathbb{R}^{2}\right)$. Also there is a Hadamard factorization of $f$, as follows. Assume that each hyperplane $\theta_{j} \cdot z=c_{j}$ in the zero set of $f$ is listed with its corresponding multiplicity. That is, if $f$ vanishes to order $p$ on the hyperplane, then the corresponding $\theta_{j}, c_{j}$ are repeated $p$ times in the sequences $\left\{\theta_{j}\right\},\left\{c_{j}\right\}$, $j=1,2, \ldots$.

Lemma 1. For some $a=\left(a_{1}, a_{2}\right) \in \mathbb{C}^{2}$, we have

$$
f(z)=\exp (a \cdot z) \prod_{j=1}^{\infty} \ell_{j}(z) \exp \left[\left(\theta_{j} \cdot z\right) / c_{j}\right] .
$$

Proof. The proof may be given by essentially repeating the proof of Hadamard's factorization theorem in one variable (it is also a consequence of P. LELONG'S representation for entire functions of finite order [8], p. 394). The main point is to show that the infinite product is an entire function (of exponential type, or even just of order 1) with the same zeros as $f$, so their quotient must be an entire function of order 1 with no zeros; i.e. $\exp (a \cdot z)$ for some $a \in \mathbb{C}^{2}$.

The next lemma allows us to reduce the theorem to the case when $I_{\text {loc }}=\widehat{\mathscr{E}}^{\prime}\left(\mathbb{R}^{2}\right)$.

Lemma 2. Let $J=\left\{h \in \hat{\mathscr{E}}^{\prime}\left(\mathbb{R}^{2}\right): h \cdot I_{\text {loc }} \subset I\right\}$. Then

(i) $J$ is a closed ideal in $\hat{\mathscr{E}}^{\prime}\left(\mathbb{R}^{2}\right)$;

(ii) $J \supset I$;

(iii) $J=\widehat{\mathscr{E}}^{\prime}\left(\mathbb{R}^{2}\right) \Leftrightarrow I=I_{10 c}$;

(iv) $J_{\text {loc }}=\hat{\mathscr{E}}^{\prime}\left(\mathbb{R}^{2}\right)$.

Proof. The assertions (i)-(iii) are all routine to check. To prove (iv), let $z_{0} \in \mathbb{C}^{2}$. We construct $h \in \hat{\mathscr{E}}^{\prime}\left(\mathbb{R}^{2}\right)$ with $h\left(z_{0}\right) \neq 0$ and $h \cdot I_{\mathrm{loc}} \subset I$. To this end, consider the (finitely many) factors of $f$ given in (7) which vanish at $z_{0}$. After relabeling, we may assume that $\ell_{1}, \ldots, \ell_{m}$ are the ones of these factors which divide every $g \in I$, listed with corresponding multiplicities (if there are no such factors, this step can be omitted). Define

$$
p(z)=\prod_{j=1}^{m} \ell_{j}(z) \quad(p \equiv 1 \text { if there are no factors })
$$

If $g \in \hat{\mathscr{E}}^{\prime}\left(\mathbb{R}^{2}\right)$ and $p$ divides $g$ near $z_{0}$, then $g / p$ is entire and $g / p \in \hat{\mathscr{E}}^{\prime}\left(\mathbb{R}^{2}\right)$.

Consider the ideal $I^{*}=(1 / p) I=\{g / p: g \in I\}$ in $\hat{\mathscr{E}}^{\prime}\left(\mathbb{R}^{2}\right)$. We claim that $z_{0}$ is at most an isolated common zero of $I^{*}$. If not, then the common zeros of the functions in $I^{*}$ near $z_{0}$ must be a variety of dimension 1 and codimension 1 . Thus there exists an analytic function $\gamma$ near $z_{0}$ such that $\gamma\left(z_{0}\right)=0$ and $\gamma$ divides every function in $I^{*}$. Then $\gamma$ also divides $f / p \in I^{*}$. Hence $\gamma$ must be, up to a factor which is nonvanishing near $z_{0}$, a product of the functions in (7). We may therefore assume that 
$\gamma$ is a product of finitely many of the factors $\ell_{j}$ which vanish at $z_{0}$. Then $G / \gamma \in \widehat{\mathscr{C}}\left(\mathbb{R}^{2}\right)$ for every $G \in I^{*}$, so actually $\gamma p$ divides every $g \in I$, contrary to the definition of $p$. Thus no such $\gamma$ exists, and $z_{0}$ is at most an isolated common zero of the functions in $1^{*}$.

We next use Theorem 2.7 of [6], p. 228. According to this result, there exists an $h \in \hat{\mathscr{E}}^{\prime}\left(\mathbb{R}^{2}\right)$ such that $h\left(z_{0}\right) \neq 0$ and $h\left[I^{*}\right]_{\text {loc }} \subset I^{*}$. But $\left[I^{*}\right]_{\text {loc }}=(1 / p) I_{\text {loc }}$, so $h I_{\mathrm{loc}}=h p\left[I^{*}\right]_{\mathrm{loc}} \subset p I^{*}=I$. Thus $h \in J$, which completes the proof of the lemma.

Lemma 3. Let $I, f$ be as in Theorem 3. If also $I_{10 c}=\hat{\mathscr{O}}\left(\mathbb{R}^{2}\right)$, then

$$
f(z) / \ell_{j}(z) \in I, \quad j=1,2, \ldots
$$

Proof. We drop the subscript $j$ throughout the proof. Let $V=\left\{z \in \mathbb{C}^{2}: \ell(z)=0\right\}$. Let $i: V \rightarrow \mathbb{C}^{2}$ be the inclusion mapping, so that $i^{*} g=g \circ i$ restricts functions on $\mathbb{C}^{2}$ to $V$. Then from Theorem 4.1 of [2], p. 98, or Theorem 4.4 .3 of [5], p. 94, we see that $i^{*} \hat{\mathscr{E}}\left(\mathbb{R}^{2}\right)$ is equal to the set of all analytic functions $\gamma$ on $V$ such that for some $A, B>0$ we have

$$
|\gamma(z)| \leqq A \exp (B p(z)), \quad z \in V,
$$

where $p(z)=\log \left(1+|z|^{2}\right)+|\operatorname{Im} z|$. It is also easy to see from the growth condition for $\gamma$ that $i^{*} \mathscr{E}^{\prime}\left(\mathbb{R}^{2}\right)$ is isomorphic to either the ring of entire functions of exponential type in one complex variable, or to $\hat{\mathscr{E}}(\mathbb{R})=\hat{\mathscr{E}}^{\prime}\left(\mathbb{R}^{1}\right)$. That is, if we make a suitable affine change of coordinates $\left(z_{1}, z_{2}\right) \leftrightarrow\left(\zeta_{1}, \zeta_{2}\right)$, for example,

$$
\begin{aligned}
& \zeta_{1}=\ell(z)=a z_{1}+b z_{2}+c \\
& \zeta_{2}=-\bar{b} z_{1}+\bar{a} z_{2},
\end{aligned}
$$

then $V$ has the equation $\zeta_{1}=0$ and the functions $i^{*} g$ are functions of $\zeta_{2}$. Since $\left(|a|^{2}+|b|^{2}\right) z_{1}=a \zeta_{1}-b \zeta_{2}-\bar{a} c$ and $\left(|a|^{2}+|b|^{2}\right) z_{2}=\bar{b} \zeta_{1}+a \zeta_{2}-\bar{b} c$, we see that $\left(i^{*} p\right)\left(\zeta_{2}\right)$ is bounded above and below by a constant times

$$
\log \left(1+\left|\zeta_{2}\right|\right)+\left|\operatorname{Im} b \zeta_{2}\right|+\left|\operatorname{Im} a \zeta_{2}\right|
$$

If $a / b$ is real, then $i^{*} \hat{\mathscr{E}}\left(\mathbb{R}^{2}\right)$ is isomorphic to $\hat{\mathscr{E}}^{\prime}(\mathbb{R})$. If $a / b$ is not real, then $i^{*} p\left(\zeta_{2}\right)$ is bounded above and below by a constant multiple of $\left|\zeta_{2}\right|$, so that $i^{*} \hat{\mathscr{E}}^{\prime}\left(\mathbb{R}^{2}\right)$ is isomorphic to the space of entire functions of exponential type in one complex variable.

In either case, it is known that, for anv ideal $\tilde{I}$ in $i^{*} \hat{\mathscr{E}}\left(\mathbb{R}^{2}\right)$, we have $\gamma \in \tilde{I}_{\text {loc }}$ if and only if there exists a sequence of functions $\gamma_{n} \in \tilde{I}$ such that $\gamma_{n} \rightarrow \gamma$ in $i^{*} \widehat{\mathscr{E}}^{\prime}\left(\mathbb{R}^{2}\right)$ (see e.g. [3] or [9]). This means that $\gamma_{n} \rightarrow \gamma$ uniformly on compact subsets of $V$ and

$$
\left|\gamma_{n}(z)\right| \leqq A \exp (B p(z)), \quad z \in V,
$$

for some constants $A, B$ independent of $n$. To complete the proof of the lemma, we have from $I_{\text {loc }}=\hat{\mathscr{E}}^{\prime}\left(\mathbb{R}^{2}\right)$ that $\left[i^{*} I\right]_{\mathrm{loc}}=i^{*} \hat{\mathscr{E}}^{\prime}\left(\mathbb{R}^{2}\right)$, so $1 \in\left[i^{*} I\right]_{\mathrm{loc}}$. Thus there exists a $g_{n} \in I$ such that $\gamma_{n}=i^{*} g_{n}$ converges to 1 in $i^{*} \widehat{\mathscr{O}}\left(\mathbb{R}^{2}\right)$. In particular, the estimate (9) holds. Then, again by Theorem 4.1 of [2], p.98, or Theorem 4.4 .3 of [5], p. 94, there exists an $h_{n} \in \mathscr{\mathscr { E }}^{\prime}\left(\mathbb{R}^{2}\right)$ such that $i^{*} h_{n}=\gamma_{n}$ and

$$
\left|h_{n}(z)\right| \leqq A^{\prime} \exp \left(B^{\prime} p(z)\right), \quad z \in \mathbb{C}^{2},
$$


and some $A^{\prime}, B^{\prime}$ independent of $n$. After possibly passing to a subsequence, we may assume $h_{n} \rightarrow h$ in $\widehat{\mathscr{E}}\left(\mathbb{R}^{2}\right)$. Then $i^{*} h=\lim _{n \rightarrow \infty} i^{*} h_{n}=1$, so $\ell(z)$ divides $1-h$ in $\mathscr{E}^{\prime}\left(\mathbb{R}^{2}\right)$. Thus

$$
f / \ell=[(1-h) / \ell] \cdot f+h(f / \ell) \in I+h f / \ell .
$$

Therefore $f / \ell \in I$ if $h f / \ell \in I$. However, $h f / \ell=\lim _{n} h_{n} f / \ell$ and $I$ is closed, so we need only prove $h_{n} f / \ell \in I$. Since

$$
h_{n} f / \ell=\left[\left(h_{n}-g_{n}\right) / \ell\right] f+g_{n}[f / \ell]
$$

and $g_{n} \in I, f \in I$, and $\left(h_{n}-g_{n}\right) / \ell \in \hat{\mathscr{C}}^{\prime}\left(\mathbb{R}^{2}\right)$, we see that $h_{n} f / \ell \in I$ which completes the proof.

Lemma 4. Let $I, f$ be as in Theorem 3 , and assume also that $I_{\mathrm{loc}}=\widehat{\mathscr{E}}^{\prime}\left(\mathbb{R}^{2}\right)$. Then $I=\hat{\mathscr{E}}^{\prime}\left(\mathbb{R}^{2}\right)$.

Proof. The proof is a repetition of the main step in Schwartz's proof of the same theorem in $\hat{\mathscr{E}}^{\prime}(\mathbb{R})$; see, e.g. [12] or [10]. We will prove

$$
D^{\alpha} f \in I \quad \text { for all } \alpha=\left(\alpha_{1}, \alpha_{2}\right) \text {, }
$$

where $D^{\alpha}=i^{-\alpha_{1}-\alpha_{2}} \frac{\partial^{\alpha_{1}+\alpha_{2}}}{\partial z_{1}^{\alpha_{1}} \partial z_{2}^{\alpha_{2}}}$. To see that the lemma follows from (10), let $T \in \mathscr{E}^{\prime}\left(\mathbb{R}^{2}\right)$ be such that $\hat{T}=f$. Then $D^{\alpha} f$ is the Fourier transform of $x^{\alpha} T=x_{1}^{\alpha_{1}} x_{2}^{\alpha_{2}} T\left(x_{1}, x_{2}\right)$. It is well-known that the closure of the linear span of $x^{\alpha} T$ contains at least one $\delta$-function, say $\delta_{a}, a \in \mathbb{R}^{2}$ (see [12]). Then $\hat{\delta}_{a}=\exp (i a \cdot z)$ is in the closure of the linear span of the $D^{\alpha} f \in I$, so $\exp (i a \cdot z) \in I$ and $I=\mathscr{E}^{\prime}\left(\mathbb{R}^{2}\right)$.

We next outline the proof of (10). By Lemma 1 ,

$$
f(z)=\exp (a \cdot z) \prod_{j=1}^{\infty} \ell_{j}(z) \exp \left[\left(\theta_{j} \cdot z\right) / c_{j}\right]
$$

so by logarithmic differentiation

$$
\frac{\partial f}{\partial z_{1}}=a_{1} f+\sum_{j=1}^{\infty}\left[\theta_{j 1} / c_{j}^{2}\right] \cdot\left[\theta_{j} \cdot z\right]\left[f(z) / \ell_{j}(z)\right] .
$$

Now each term on the right hand side of (11) belongs to $I$ by Lemma 3. Furthermore, by the argument given in $[10]$, the series converges in $\hat{\mathscr{E}}^{\prime}\left(\mathbb{R}^{2}\right)$. Therefore $\partial f / \partial z_{1} \in I$, and similarly $\partial f / \partial z_{2} \in I$.

To see that $D^{\alpha} f \in I$ for higher order derivatives of $f$, differentiate both sides of (11) the appropriate number of times. The series converges to $D^{\alpha} f$ in $\hat{\mathscr{E}}^{\prime}\left(\mathbb{R}^{2}\right)$. Each term $f_{j}(z)=\left(\theta_{j} \cdot z\right) f(z) / \ell_{j}(z)$ belongs to $I$ and its zero set is a union of hyperplanes. Thus, $\partial f_{j} / \partial z_{i} \in I$ by what we just proved. Therefore all second order derivatives of $f$ belong to $I$. Continuing in this fashion, the proof of $(10)$ is concluded.

Proof of Theorem 3. By (iv) and (ii) of Lemma 2, and by Lemma 4, we have $J=\hat{\mathscr{E}}^{\prime}\left(\mathbb{R}^{2}\right)$. Hence, by (iii) of Lemma 2 , we see that $I=I_{\mathrm{loc}}$.

Remarks. In the case when $n \geqq 3$ and $I_{\text {loc }}=\hat{\mathscr{E}}^{\prime}\left(\mathbb{R}^{n}\right)$, the same proof will work, except that we must assume that in each linear variety $V$ the constant function 1 is a limit of functions $g_{n} \in I$. When $n=3$, for example, this will be the case if $i^{*}(I)$ 
[with $i: V \rightarrow \mathbb{C}^{3}$ ] contains a function whose zero set in $V$ is a union of hyperplanes. Thus, if we assume

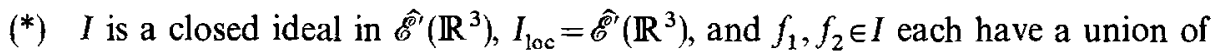
hyperplanes as their zero sets,

and if further $f_{i}$ is not $\equiv 0$ on any hyperplane on which $f_{j}=0, i \neq j$, then $I=\hat{\mathscr{E}}\left(\mathbb{R}^{3}\right)$. By induction, then, and a slight modification of Lemma 2 , we obtain the following result.

Theorem 4. Let 1 be a closed ideal in $\tilde{\mathscr{O}}\left(\mathbb{R}^{n}\right)$ which contains functions $f_{1}, \ldots, f_{N}$ such that

(i) the zero set of each $f_{i}$ is a union of hyperplanes, and

(ii) the set of common zeros of $f_{1}, \ldots, f_{N}$ is discrete.

Then $I=I_{\text {loc }}$.

Note. This work was supported in part by NSF Grants MPS $75-06977$ and GP 67328 .

\section{Bibliography}

1. Brown, L., B.M. Schreiber \& B.A. TAYlor, Spectral synthesis and the Pompeiu problem, Ann. Inst. Fourier (Grenoble) 23, 125-154 (1973).

2. Ehrenpreis, L., Fourier Analysis in Several Complex Variables. New York: Wiley-Interscience 1970.

3. Ehrenpreis, L., Mean periodic functions, Amer. J. Math. 76, 293 -328 (1966).

4. Gurevich, D.I., Counterexamples to a problem of L. Schwartz, Funct. Anal. Appl., 197, 116-120 (1975).

5. Hörmander, L., An Introduction to Complex Analysis in Several Variables. Princeton: Van Nostrand 1966.

6. Kelleher, J.J. \& B.A. TAYLOR, Finitely generated ideals in rings of analytic functions, Math. Ann. 193, 225-237 (1971)

7. Kelleher, J.J. \& B.A. TAYLOR, Closed ideals in locally convex algebras of analytic functions. J. Reine Angew. Math. 255, 190-209 (1972).

8. LELONG, P., Fonctions entières et fonctions plurisous-harmoniquesd'ordre finidans $\mathbb{C}^{n}$, J. d'Analyse Math. 12, 365-407 (1964).

9. Malgrange, B., Existence et approximation des solutions des équations aux derivées partielles et des équations de convolution, Ann. de l'Inst. Fourier (Grenoble) 6, 271-355 (1916).

10. Malgrange, B., Sur les équations de convolution, Univ. Torino Rend. Sem. Math. 19, 19-27 $(1959 / 60)$.

11. Schwartz, L., Théorie des Distributions. Paris: Hermann 1950.

12. Schwartz, L., Théorie générale des fonctions moyenne-periodiques, Ann. of Math. 48, 857-928 (1947).

13. Zalcman, L., Analyticity and the Pompeiu problem, Arch. Rational Mech. Anal. 47, 237-254 (1972).

14. ZaLCMAN, L., Mean values and differential equations, Israel I. Math. 14, 339-352 (1973).

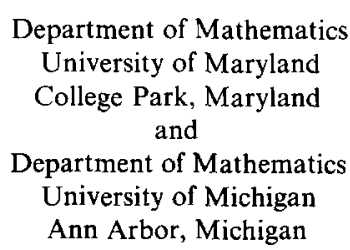

